\title{
Exigência de lisina para mantença determinada com galos de diferentes genótipos
}

\author{
Jefferson Costa de Siqueira ${ }^{1,2}$, Nilva Kazue Sakomura ${ }^{3}$, Horacio Santiago Rostagno ${ }^{4}$, Melina \\ Aparecida Bonato ${ }^{1}$, Sandra Regina Freitas Pinheiro ${ }^{1,5}$, Dáphinne Cardoso Nagib \\ Nascimento ${ }^{1,6}$
}

\author{
1 Pós-graduação em Zootecnia - FCAV/UNESP - Jaboticabal, SP. \\ 2 Departamento de Zootecnia/Centro de Ciências Agrárias e Ambientais - CCAA/UFMA - Chapadinha, MA. \\ ${ }^{3}$ Departamento de Zootecnia/FCAVIUNESP - Jaboticabal, SP. Bolsista CNPQ e membro INCT. \\ ${ }^{4}$ Departamento de Zootecnia/UFV - Viçosa, MG \\ 5 Departamento de Zootecnia/UFVJM - Diamantina, MG. \\ ${ }^{6}$ Perdigão Agroindustrial S/A - Rio Verde, GO.
}

RESUMO - Para estimar as exigências de lisina digestível para mantença de galos adultos de diferentes genótipos e pesos corporais (Leghorn, ISA Label e Cobb 500), foram conduzidos três ensaios de metabolismo empregando-se a técnica do balanço de nitrogênio (BN). As aves foram distribuídas em delineamento inteiramente casualizado, com cinco níveis de lisina digestível e seis repetições, sendo as unidades experimentais compostas por uma (ensaios 1) ou duas aves (ensaios 2 e 3 ). Em cada ensaio, a exigência de lisina para mantença foi obtida por meio da regressão linear do balanço de nitrogênio ( $\mathrm{mg} / \mathrm{kg}^{0,75} / \mathrm{dia}$ ) pelo consumo de lisina digestível ( $\mathrm{mg} / \mathrm{kg}^{0,75} /$ dia) e definida pelo consumo de lisina digestível necessário para manter o balanço de nitrogênio igual a zero. Para comparar as respostas dos diferentes genótipos, os dados de balanço de nitrogênio obtidos nos ensaios foram analisados por teste de paralelismo, utilizando-se como variável classificatória o genótipo e como covariável o consumo de lisina digestível. A exigência de lisina digestível, quando estabelecida com base no peso metabólico, não variou entre os genótipos e foi definida pela equação geral: $\mathrm{BN}=-122,163+2,706 \mathrm{CLis}(\mathrm{P}<0,001$; $\left.r^{2}=0,67\right)$. Com base nesta equação, estimou-se a exigência de lisina digestível em 45,1 mg/kg0,75/dia (32,3 mg/kg/dia), independentemente do genótipo. Quando estabelecidas com base no conteúdo e no grau de maturidade da proteína corporal, as exigências de lisina para mantença de galos Leghorn, ISA Label e Cobb 500 são de 173,6; 164,0 e 140,9 mg/PB ${ }_{\mathrm{m}}^{0,73 . u / d i a, ~}$ respectivamente.

Palavras-chave: aminoácidos, balanço de nitrogênio, frangos de corte, método fatorial

\section{Lysine requirements for maintenance determined with roosters of different genotypes}

\begin{abstract}
To estimate the digestible lysine requirements for maintenance of roosters of different genotypes and body weight (Leghorn, ISA Label and Cobb 500), it was carried out three metabolic trials using the nitrogen balance (NB) technique. The birds were distributed in a completely randomized design with five levels of digestible lysine and six replications, and the experimental units consisted of one (trial 1) or two birds (trial 2 and 3). In each trial, the requirement of lysine for maintenance was obtained by linear regression of nitrogen balance $\left(\mathrm{mg} / \mathrm{kg}^{0.75} /\right.$ day) by digestible lysine intake ( $\mathrm{mg} / \mathrm{kg}^{0.75} /$ day), defined by the digestible lysine intake required to maintain nitrogen balance equal to zero. To compare the responses from the different genotypes, the nitrogen balance data obtained in the trials were analyzed by parallelism test, using the genotype as a classificatory variable and the digestible lysine intake as covariate. The requirement of digestible lysine did not vary among the genotypes, when it was established on the basis of metabolic weight, and was defined by the general equation: $\mathrm{NB}=-122.163+2.706$ CLYS $\left(\mathrm{P}<0.001, \mathrm{r}^{2}=0.67\right)$. Based on this equation, it was estimated the requirement of digestible lysine at $45.1 \mathrm{mg} / \mathrm{kg}^{0,75} /$ day $(32.3 \mathrm{mg} / \mathrm{kg} /$ day), regardless to the genotype. When they are established based on the content and degree of maturity of body protein, the lysine requirements are 173.6, 164.0 and $140.9 \mathrm{mg} / \mathrm{PB}_{\mathrm{m}}{ }^{0.73} . \mathrm{u} / \mathrm{day}$ for roosters Leghorn, ISA Label and Cobb 500, respectively
\end{abstract}

Key Words: amino acids, broiler chickens, factorial approach, nitrogen balance 


\section{Introdução}

A mantença pode ser definida como o estado de equilíbrio no qual a ingestão de nitrogênio é igual ao somatório das perdas e o conteúdo de nitrogênio corporal permanece constante(Owens \& Pettigrew, 1989; Fisher, 1998; Sakomura \& Coon, 2003; Sakomura \& Rostagno, 2007).

As exigências de lisina para a mantença foram estudadas com aves em crescimento (Hruby, 1998; Edward et al., 1999) e adultas (Leville \& Fisher, 1959a; Sakomura \& Coon, 2003; Nonis \& Gous, 2008), entretanto, os resultados das pesquisas divergem amplamente, sendo este cenário parcialmente atribuído à utilização de diferentes métodos experimentais (Sakomura \& Rostagno, 2007), motivando permanentes discussões na esfera científica.

De acordo com Owens \& Pettigrew (1989), nos estudos para estimar as exigências de aminoácidos para mantença podem ser utilizados animais em crescimento ou adultos, relacionando a ingestão do aminoácido-teste com a retenção de nitrogênio corporal, por meio da técnica do abate comparativo ou do balanço de nitrogênio. Contudo, Burnham \& Gous (1992) advertem que a interpretação dos resultados de experimentos com animais em crescimento deve ser cautelosa, em razão das modificações na taxa de crescimento e na proporção do aminoácido consumido que será utilizada para mantença durante o período experimental. Assim, a determinação com aves adultas improdutivas mostra-se mais adequada, uma vez que as exigências dessas aves estão associadas apenas às perdas inevitáveis (mantença), não incluindo necessidades específicas de aminoácidos para o crescimento e/ou a produção (Owens \& Pettigrew, 1989).

Outro aspecto de relevância está relacionado à unidade para expressar as exigências de mantença. A maioria dos estudos relaciona as exigências de aminoácidos para mantença ao peso da ave em jejum (mg/kg/dia) ou ao peso metabólico (mg/kg0,75/dia). Entretanto, Emmans \& Fisher (1986) demonstraram que as exigências de aminoácidos para mantença estão mais intimamente relacionadas ao conteúdo de proteína corporal, tendo em vista a inexistência de demanda de aminoácidos para manutenção das reservas lipídicas (Emmans \& Oldham, 1988; Burnham \& Gous, 1992; Gous, 2007) e são, portanto, a forma mais apropriada para expressar as exigências, uma vez que o conteúdo de lipídios pode variar até mesmo entre aves com pesos corporais semelhantes (Emmans \& Fisher, 1986; Sakomura \& Coon, 2003; Nonis \& Gous, 2008).

Com base nessas informações, realizou-se este estudo com o objetivo de estimar as exigências de lisina digestível para mantença de galos de diferentes genótipos (Leghorn,
ISA Label e Cobb 500), com base nos pesos corporal, metabólico e proteico.

\section{Material e Métodos}

Foram conduzidos três ensaios de metabolismo para quantificar o balanço de nitrogênio de galos adultos (idade igual ou superior a 27 semanas), no Setor de Avicultura do Departamento de Zootecnia da Faculdade de Ciências Agrárias e Veterinárias - FCAV / UNESP, Jaboticabal - SP, objetivando determinar as exigências de lisina digestível para mantença de aves de diferentes genótipos e composições corporais à maturidade. Os estudos foram realizados de forma independente e em ordem cronológica.

Em cada ensaio, as aves foram distribuídas em gaiolas metabólicas de arame galvanizado $\left(0,20 \mathrm{~m}^{2}\right)$, equipadas com bebedouro tipo nipple e comedouro do tipo calha, em delineamento experimental inteiramente casualizado, com cinco níveis de lisina e seis repetições. No ensaio 1 as unidades experimentais foram compostas por uma ave, utilizando-se 30 galos Leghorn, enquanto nos ensaios 2 e 3 as unidades experimentais foram compostas por duas aves, utilizando-se 60 galos ISA Label (ensaio 2) ou Cobb 500 (ensaio 3). No início de cada ensaio as aves foram pesadas individualmente possibilitando a constituição de parcelas homogêneas.

Em todos os ensaios, as aves tiveram acesso às dietas experimentais durante todo o período experimental, cuja duração foi de 9 dias ( 5 de adaptação às dietas experimentais e 4 para a coleta total das excretas).

No ensaio 1, com galos Leghorn, as dietas experimentais foram obtidas à partir da diluição de uma dieta concentrada contendo $0,179 \%$ de lisina digestível, à base de milho e amido de milho, com outra isenta de lisina, à base de amido de milho (Tabela 1), resultando em dietas isoenergéticas e isonitrogênicas contendo 0,036; 0,071; 0,107; 0,143 e 0,179\% de lisina digestível (Tabela 2).

As concentrações dos nutrientes nas dietas foram estabelecidas considerando um consumo médio diário de 120 g de ração, assumindo o atendimento de pelo menos $50 \%$ das exigências de energia e aminoácidos essenciais (exceto lisina), 100\% das exigências de minerais e vitaminas, tomando-se como referência as recomendações de Rostagno et al. (2005) para galos. No final do período experimental, as sobras de ração foram quantificadas.

Tendo em vista a variação no consumo das dietas experimentais durante o ensaio 1 , as dietas utilizadas nos ensaios 2 e 3 foram mais concentradas que aquela utilizada no ensaio 1 e foram obtidas pela mistura de uma dieta 
contendo 0,093\% de lisina digestível (basal) com outra contendo 0,464\% (concentrada) (Tabela 3), em diferentes proporções, resultando em dietas isonutritivas contendo 0,093; 0,186; 0,278; 0,371 e 0,464\% de lisina digestível (Tabela 4). Nestes ensaios o fornecimento das dietas foi controlado pelo peso metabólico das aves ( $\left.20 \mathrm{~g} / \mathrm{kg}^{0,75} / \mathrm{dia}\right)$, permitindo que os galos ISA Label (ensaio 2) e Cobb 500 (ensaio 3) consumissem todo o alimento ofertado, contribuindo para a redução do erro experimental.

Para delimitar o início e o final do período de coleta das excretas, utilizou-se óxido de ferro como marcador, adicionado nas dietas experimentais na concentração de $1 \%$. As excretas não marcadas na primeira coleta e as

Tabela 1 - Composição das dietas utilizadas no ensaio 1, com galos Leghorn

\begin{tabular}{|c|c|c|}
\hline \multirow[t]{2}{*}{ Ingrediente (\%) } & \multicolumn{2}{|c|}{ Dieta } \\
\hline & Isenta de lisina & Concentrada \\
\hline Milho & - & 49,863 \\
\hline Amido de milho & 80,376 & 39,490 \\
\hline Casca de arroz & 9,987 & 7,406 \\
\hline Fosfato bicálcico & 1,226 & 1,016 \\
\hline Calcário calcítico & 0,285 & 0,441 \\
\hline Cloreto de sódio & 0,418 & 0,400 \\
\hline Óleo de soja & 0,600 & 0,800 \\
\hline DL-metionina (99\%) & 0,192 & 0,024 \\
\hline L-lisina $\mathrm{HCl}(78,5 \%)$ & - & 0,095 \\
\hline L-treonina (99\%) & 0,178 & 0,040 \\
\hline L-triptofano (99\%) & 0,053 & 0,022 \\
\hline L-arginina (99\%) & 0,254 & 0,072 \\
\hline L-valina (99\%) & 0,228 & 0,052 \\
\hline L-isoleucina (99\%) & 0,140 & 0,009 \\
\hline L-leucina (99\%) & 0,491 & - \\
\hline L-fenilalanina (99\%) & 0,190 & - \\
\hline Suplemento vitamínico ${ }^{1}$ & 0,100 & 0,100 \\
\hline Suplemento mineral ${ }^{2}$ & 0,100 & 0,100 \\
\hline Cloreto de colina $(70 \%)$ & 0,070 & 0,070 \\
\hline Ácido L-glutâmico (99\%) & 5,112 & - \\
\hline \multicolumn{3}{|c|}{$\begin{array}{l}{ }^{1} \text { Conteúdo/kg: vit. A - } 12.000 .000 \text { UI; vit. D3 - } 22.000 .000 \text { UI; vit. E - } 30.000 \text { mg; } \\
\text { vit. B1 - 2.200 mg; vit. B2 - } 6.000 \text { mg; vit. B6 - } 3.300 \text { mg; vit. B12 - } 16.000 \text { mg; } \\
\text { niacina - } 53.000 \mathrm{mg} \text {; ácido pantotênico - } 13.000 \mathrm{mg} \text {; vit. K - 2.500 mg; ácido } \\
\text { fólico - } 1.000 \text { mg; Se - } 250 \mathrm{mg} \text {; antioxidante - } 100.000 \mathrm{mg} \text {; veículo qsp - } 1.000 \text { g. } \\
2 \text { Conteúdo/kg: Mn - } 75.000 \text { mg; Fe - } 50.000 \text { mg; Zn - } 70.000 \text { mg; Cu - } 6.500 \text { mg; } \\
\text { Co - } 200 \text { mg; I - } 1.500 \text { mg; veículo qsp - } 1.000 \text { g. }\end{array}$} \\
\hline
\end{tabular}

marcadas na última coleta foram descartadas. As excretas foram coletadas duas vezes por dia (às 8 h e às 16 h), após remoção de contaminantes (penas e descamações), utilizando-se bandejas revestidas por lona plástica dispostas sob as gaiolas metabólicas. As excretas produzidas por cada unidade experimental durante cada período de coleta foram acondicionadas em sacos plásticos devidamente identificados e congeladas $\left(-20^{\circ} \mathrm{C}\right)$ para análises posteriores.

As excretas produzidas por cada unidade experimental, em cada ensaio de metabolismo, foram descongeladas em temperatura ambiente, pesadas e homogeneizadas com o uso de um liquidificador doméstico (RI2008, Walita ${ }^{\circledR}$ ) adicionando-se um volume conhecido de água destilada para obter uma consistência adequada, quando necessário, conforme descrito por Burnham \& Gous (1992). Nesse caso, o peso correspondente ao volume de água destilada adicionado foi somado ao total de excretas produzidas pela parcela.

Do total de excretas referente a cada parcela, foram retiradas alíquotas, que foram acondicionadas em placas de Petri descartáveis, pesadas e congeladas novamente $\left(-20^{\circ} \mathrm{C}\right)$, até a pré-secagem. Para a obtenção da matéria préseca as amostras foram liofilizadas por 72 horas $\left(-50^{\circ} \mathrm{C}\right.$; $-80 \mathrm{kPa})\left(\mathrm{VLP} 20\right.$, Thermo ${ }^{\circledR}$ ) e pesadas novamente, sendo na seqüência, processadas em moinho de bola (MA-350, Marconi ${ }^{\circledR}$ ) por $2 \mathrm{mim}$. As dietas experimentais foram processadas da mesma forma, sendo encaminhadas ao laboratório, juntamente com as excretas, para determinação dos teores de matéria seca e nitrogênio total.

A secagem definitiva das dietas e das excretas foi realizada em estufa $\left(\right.$ Químis ${ }^{\circledR}$ ) a $105^{\circ} \mathrm{C}$ durante 4 horas. Para a determinação do teor de nitrogênio total das amostras utilizou-se o método de Kjeldahl (AOAC, 1995).

Em todos os ensaios o balanço de nitrogênio (BN) foi determinado pela diferença entre a ingestão (mg/kg0,75/dia) e a excreção de nitrogênio ( $\mathrm{mg} / \mathrm{kg}^{0,75} /$ dia) das aves submetidas à ingestões crescentes de lisina digestível. A

Tabela 2 - Níveis de lisina das dietas utilizadas no ensaio 1, com galos Leghorn

\begin{tabular}{|c|c|c|c|c|c|}
\hline \multirow[t]{2}{*}{ Dieta (\%) } & \multicolumn{5}{|c|}{ Nível de lisina digestível (\%) } \\
\hline & 0,036 & 0,071 & 0,107 & 0,143 & 0,179 \\
\hline Concentrada & 20,11 & 39,67 & 59,78 & 79,89 & 100,000 \\
\hline Total & 100,00 & 100,00 & 100,00 & 100,00 & 100,00 \\
\hline \multicolumn{6}{|c|}{ Composição nutricional ${ }^{1}$} \\
\hline Proteína bruta (\%) & $4,60(5,22)^{2}$ & $4,60(5,28)$ & $4,60 \quad(5,27)$ & $4,60 \quad(5,31)$ & $4,60(5,42)$ \\
\hline
\end{tabular}


exigência de lisina para a mantença das aves foi considerada como a quantidade de lisina digestível ingerida ( $\mathrm{mg} / \mathrm{kg}^{0,75} / \mathrm{dia}$ ) capaz de proporcionar balanço de nitrogênio igual a zero, conforme descrito por Sakomura \& Rostagno (2007).

Para determinar as exigências de mantença em função do peso de proteína do corpo depenado, no final de cada ensaio foram abatidos dois galos, que foram processados, e posteriormente quantificados os teores de proteína bruta corporal (AOAC, 1995). Utilizou-se a equação proposta por Emmans \& Fisher (1986): PM = 0,008.PB ${ }_{\mathrm{m}}{ }^{0,73}$.u; em que: $\mathrm{PM}=$ exigência de proteína para a mantença ( $\mathrm{kg} / \mathrm{dia})$; 0,008 = constante ( $\mathrm{kg}$ de proteína ideal por unidade de mantença, $\mathrm{P}_{\mathrm{m}}{ }^{0,73} \cdot \mathrm{u}$ ), definida com base na pressuposição que as aves exigem $8 \mathrm{~g}$ de uma proteína com composição

Tabela 3 - Composição das dietas utilizadas nos ensaios 2 e 3

\begin{tabular}{|c|c|c|}
\hline \multirow[t]{2}{*}{ Ingrediente (\%) } & \multicolumn{2}{|c|}{ Dieta } \\
\hline & Basal & Concentrada \\
\hline Milho & 44,286 & 74,124 \\
\hline Amido de milho & 22,134 & 10,650 \\
\hline Casca de arroz & 9,396 & - \\
\hline Fosfato bicálcico & 3,030 & 2,923 \\
\hline Calcário calcítico & 1,197 & 1,463 \\
\hline Óleo de soja & 12,296 & 7,004 \\
\hline Cloreto de sódio & 1,110 & 1,121 \\
\hline DL-metionina (99\%) & 0,348 & 0,247 \\
\hline L-lisina $\mathrm{HCl}(78,5 \%)$ & - & 0,393 \\
\hline L-treonina (99\%) & 0,340 & 0,257 \\
\hline L-triptofano (99\%) & 0,110 & 0,092 \\
\hline L-arginina (99\%) & 0,496 & 0,387 \\
\hline L-valina (99\%) & 0,438 & 0,333 \\
\hline L-isoleucina (99\%) & 0,395 & 0,316 \\
\hline L-leucina $(99 \%)$ & 0,294 & - \\
\hline L-fenilalanina (99\%) & 0,224 & 0,110 \\
\hline Suplemento vitamínico ${ }^{1}$ & 0,200 & 0,200 \\
\hline Suplemento mineral ${ }^{2}$ & 0,200 & 0,200 \\
\hline Cloreto de colina (70\%) & 0,180 & 0,180 \\
\hline Ácido L-glutâmico (99\%) & 3,326 & - \\
\hline \multicolumn{3}{|c|}{ 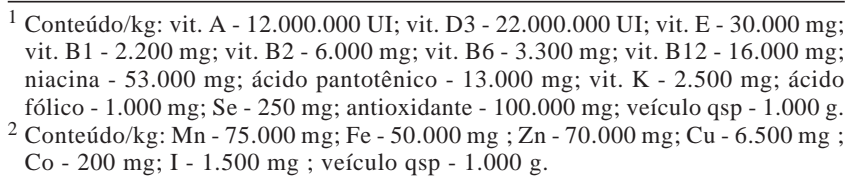 } \\
\hline
\end{tabular}

adequada para suprir as necessidades de mantença; $\mathrm{PB}_{\mathrm{m}}=$ peso de proteína à maturidade $(\mathrm{kg})$; e u = grau de maturidade da proteína corporal no tempo $\mathrm{t}\left(\mathrm{u}=\mathrm{PB}_{\mathrm{t}} / \mathrm{PB}_{\mathrm{m}}\right)$, em que $\mathrm{u}=1$ para aves adultas ou $\mathrm{u}<1$ para aves em crescimento. Desse modo, as exigências diárias de lisina para mantença também foram expressas como $\mathrm{mg} / \mathrm{PB}_{\mathrm{m}}{ }^{0,73}$.u/dia.

Os dados de cada ensaio foram submetidos à análises de regressão, considerando o consumo de lisina digestível ( $\mathrm{mg} / \mathrm{kg}^{0,75} / \mathrm{dia}$ ) como variável independente e o balanço de nitrogênio ( $\mathrm{mg} / \mathrm{kg}^{0,75} / \mathrm{dia}$ ) como dependente (BN), segundo o modelo: $\mathrm{BN}=\beta_{0}+\beta_{1}$ CLys $+\varepsilon$; em que $\beta_{0}$ e $\beta_{1}$ são os parâmetros da regressão e $\varepsilon$ é o erro aleatório. Para verificar o ajuste das equações considerou-se o nível de significância do teste $\mathrm{F}$ sob as hipóteses $\mathrm{H}_{0}$ : $\beta_{1}=0$ e $\mathrm{H}_{1}$ : $\beta_{1} \neq 0$. As exigências de lisina para a mantença foram obtidas utilizando-se a expressão: CLys $=\beta_{0} / \beta_{1}$.

Para comparar as respostas dos diferentes genótipos, os dados de balanço de nitrogênio obtidos nos ensaios foram analisados por teste de paralelismo, utilizando-se como variável classificatória o genótipo (G) e como co-variável o consumo de lisina digestível (CLys), segundo o modelo descrito por Kaps \& Lamberson (2004): $\mathrm{BN}_{i j}=\beta_{0}+\mathrm{G}_{i}+$ $\beta_{1} \mathrm{CLys}_{i j}+\Sigma_{i} \beta_{2 i}$ (G*CLys $_{i j}+\varepsilon_{i j}$; em que $\mathrm{BN}_{i j}=$ balanço de nitrogênio correspondente à observação $\mathrm{j}$ do genótipo $\mathrm{i}$; $\mathrm{G}_{\mathrm{i}}=$ efeito do genótipo; $\beta_{0}, \beta_{1}$ e $\beta_{2 \mathrm{i}}=$ parâmetros da regressão; $\left(\mathrm{G}^{*} \mathrm{CLys}\right)_{i j}=$ efeito da interação entre a variável classificatória e a covariável; e $\varepsilon_{i j}=$ erro aleatório associado a observação j do genótipo i. Neste caso, as hipóteses testadas foram:

a) $H_{0}$ : $G_{i}=0$ para todo $i$, não existe efeito de genótipo.

$\mathrm{H}_{1}$ : $\mathrm{Gi} \neq 0$ para pelo menos um $\mathrm{i}$; existe efeito de genótipo.

b) $\mathrm{H}_{0}: \beta_{1}=0$, a inclinação geral é igual à zero, não existe regressão.

$\mathrm{H}_{1}: \beta_{1} \neq 0$, a inclinação geral difere de zero, existe uma regressão.

c) $\mathrm{H}_{0}: \beta_{2 \mathrm{i}}=0$, a inclinação do genótipo i não difere da inclinação média.

Tabela 4 - Níveis de lisina digestível nas dietas utilizadas nos ensaios 2 e 3

\begin{tabular}{|c|c|c|c|c|c|}
\hline \multirow[t]{2}{*}{ Dieta (\%) } & \multicolumn{5}{|c|}{ Nível de lisina digestível (\%) } \\
\hline & 0,093 & 0,186 & 0,278 & 0,371 & 0,464 \\
\hline Concentrada & - & 25,00 & 50,00 & 75,00 & 100,00 \\
\hline Total & 100,00 & 100,00 & 100,00 & 100,00 & 100,00 \\
\hline \multicolumn{6}{|c|}{ Composição nutricional ${ }^{1}$} \\
\hline Proteína bruta (\%) & $8,12(8,32)^{2}$ & $8,12(8,12)$ & $8,12(8,46)$ & $8,12(8,50)$ & $8,12(8,16)$ \\
\hline
\end{tabular}



média.

$\mathrm{H}_{1}: \beta_{2 \mathrm{i}} \neq 0$, a inclinação do genótipo i difere da inclinação

As análises estatísticas foram realizadas considerandose um nível de significância de até $5 \%$ por meio do procedimento GLM do software SAS 9.0 (2002).

\section{Resultados e Discussão}

As exigências de lisina para a mantença de aves sexualmente maduras vêm sendo estudadas há décadas (Leville \& Fisher, 1959a; Sakomura \& Coon, 2003; Nonis \& Gous, 2008), entretanto, os resultados são conflitantes, sendo este cenário atribuído à utilização de diferentes métodos experimentais e à diferentes formas de interpretação e expressão dos resultados.

A variação no peso corporal das aves durante os períodos de coleta (Tabela 5) foi semelhante àquelas observadas nos estudos de Leveille \& Fisher (1959a) e Leveille \& Fisher (1959b), cujas magnitudes não excederam -4 e -7\%, respectivamente. Estudos para determinar as exigências de mantença exigem a utilização de dietas extremamente deficientes no aminoácido teste, sendo previsível que as aves percam peso. Contudo, o peso corporal não é considerado um bom indicativo de adequação das dietas experimentais, tendo em vista que estas pequenas modificações de peso podem ser facilmente atribuídas às diferenças na ingestão de água e perda de penas durante o período experimental (Leveille \& Fisher, 1959a).

Nos três ensaios de metabolismo, os consumos de lisina abrangeram intervalos suficientes para proporcionar balanços de nitrogênio negativos, próximos a zero e positivos, possibilitando as estimativas das exigências sem que houvesse a necessidade de extrapolações (Tabela 6).

A equação que descreveu o balanço de nitrogênio (mg/kg0,75/dia) dos galos Leghorn (ensaio 1) em função do consumo de lisina (mg/kg0,75/dia) foi: $\mathrm{BN}_{\mathrm{L}}=-107,425+$ 2,392 CLis ( $\left.\mathrm{P}<0,001 ; \mathrm{r}^{2}=0,60\right)$, estimando-se as exigências de lisina para a mantença ( Lis $_{\mathrm{mL}}$ ) em 44,9 mg/kg0,75/dia $(107,425 / 2,392=44,9)$. Considerando o peso médio dos galos Leghorn utilizados no ensaio 1, de 1,99 kg, as exigências corresponderama 37,8 mg/kg/dia ((44,9*1,990,75)/1,99=37,8).

Apesar dos resultados satisfatórios obtidos no ensaio, os galos Leghorn que receberam as dietas mais deficientes, contendo 0,036; 0,071 e 0,107\%, de lisina digestível, apresentaram reduções no consumo de ração (g/ave/dia) equivalentes a 38,2; 25,1 e 18,2\%, respectivamente, em relação à dieta contendo $0,143 \%$ de lisina. Resultados semelhantes foram observados por Leveille \& Fisher (1960) e Leveille et al. (1960), que atribuíram a redução do consumo ao desbalanço natural das dietas mais deficientes. De acordo com a "teoria aminostática de regulação do consumo", relatada por Gonzales (2002), dietas com grave desbalanço de aminoácidos resultam em acentuada redução no consumo de ração. Com base nisso, é provável que o desbalanço ocasionado pela severa deficiência de lisina nessas dietas seja o principal responsável pelo menor consumo observado nos três primeiros níveis avaliados.

Outro fator de ordem prática que pode ter contribuído para a redução do consumo de ração relaciona-se com a composição das dietas experimentais. Para obtenção de

Tabela 5 - Pesos médios inicial e final e variação de peso dos galos durante os períodos de coleta

\begin{tabular}{|c|c|c|c|c|}
\hline Genótipo & Nível de lisina digestível (\%) & \multicolumn{2}{|c|}{ Peso corporal (kg) } & Variação $(\%)^{1}$ \\
\hline \multirow{4}{*}{ (1) Leghorn } & 0,036 & $1,94 \pm 0,06$ & $1,91 \pm 0,06$ & $-1,90 \pm 0,59$ \\
\hline & 0,107 & $1,91 \pm 0,06$ & $1,88 \pm 0,06$ & $-1,54 \pm 0,28$ \\
\hline & 0,143 & $2,03 \pm 0,06$ & $2,01 \pm 0,06$ & $-1,03 \pm 0,44$ \\
\hline & 0,179 & $2,06 \pm 0,09$ & $2,05 \pm 0,09$ & $0,48 \pm 0,46$ \\
\hline \multirow{4}{*}{ (2) ISA Label } & 0,186 & $4,31 \pm 0,07$ & $4,21 \pm 0,08$ & $-2,34 \pm 0,42$ \\
\hline & 0,278 & $4,32 \pm 0,07$ & $4,27 \pm 0,05$ & $-1,15 \pm 0,16$ \\
\hline & 0,371 & $4,35 \pm 0,07$ & $4,32 \pm 0,07$ & $-0,69 \pm 0,34$ \\
\hline & 0,464 & $4,40 \pm 0,06$ & $4,36 \pm 0,07$ & $-1,06 \pm 0,40$ \\
\hline \multirow{2}{*}{ (3) Cobb 500} & 0,093 & $5,40 \pm 0,31$ & $5,16 \pm 0,31$ & $-4,53 \pm 0,58$ \\
\hline & 0,186 & $5,12 \pm 0,07$ & $4,92 \pm 0,08$ & $-3,82 \pm 0,89$ \\
\hline
\end{tabular}

${ }^{1}$ (Peso final - Peso inicial) / Peso inicial $* 100$.

Os valores apresentados para o ensaio 1 correspondem à média ( \pm erro-padrão) de seis aves, enquanto os apresentados nos ensaios 2 e 3 correspondem à médias ( \pm erro-padrão) de 12 aves. 
Tabela 6 - Consumos de ração, de lisina digestível e de nitrogênio, excreção de nitrogênio e balanço de nitrogênio em galos de três genótipos

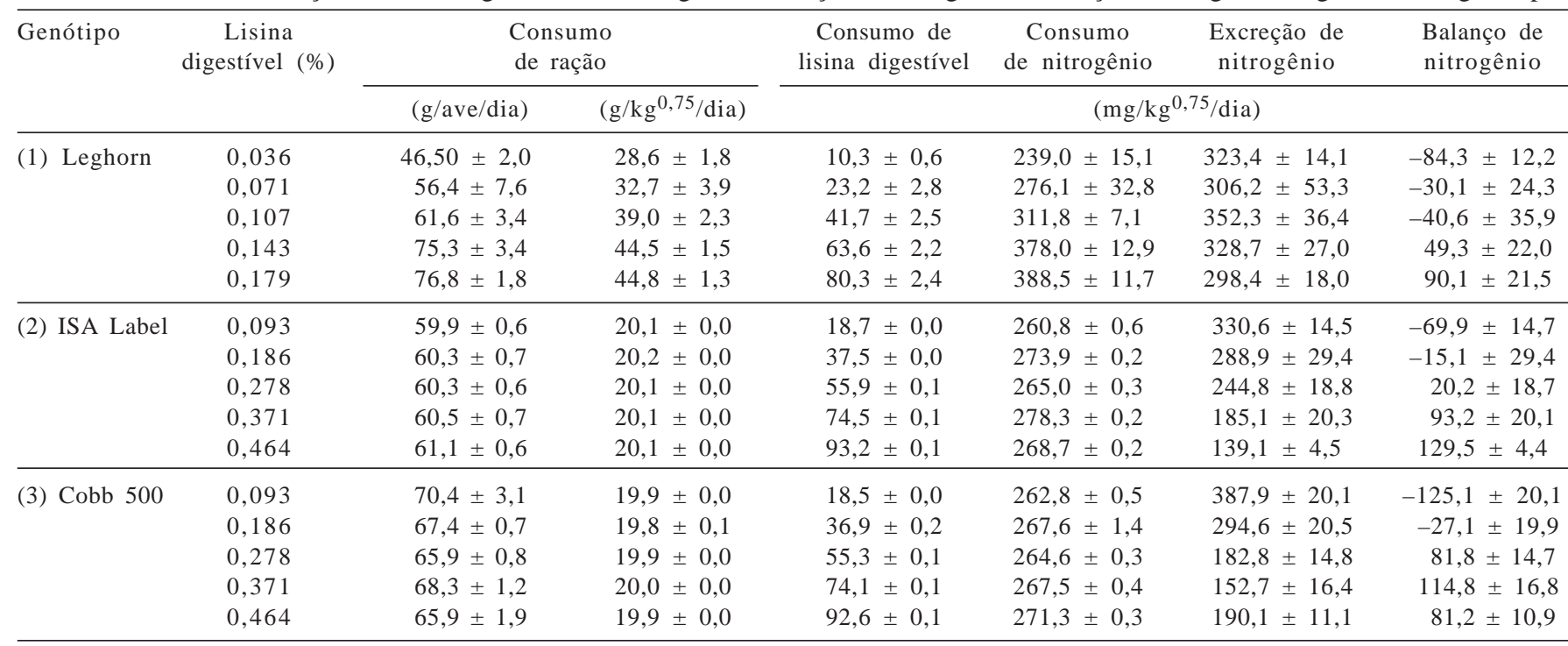

${ }^{1}$ Os valores apresentados no ensaio com galos Leghorn correspondem à médias de seis aves, enquanto os apresentados nos ensaios com Isa Label e Cobb 500 correspondem à média de 12 aves.

níveis suficientemente baixos de lisina digestível nas dietas avaliadas, utilizou-se como diluente uma dieta isenta de lisina à base de amido de milho. Considerando que as proporções de diluição da dieta concentrada variaram de 79,89 a $0 \%$, as dietas mais deficientes apresentaram maior pulverulência, o que possivelmente afetou o consumo dos galos Leghorn.

Com os objetivos de reduzir a pulverulência das dietas experimentais e controlar a variação no consumo, nos ensaios com os galos Isa Label e Cobb 500, foram utilizadas dietas mais concentradas (Tabela 4), fornecidas de acordo com o peso metabólico das aves (20 g/kg0,75/dia). Essa medida possibilitou que os galos consumissem todo o alimento ofertado, contribuindo para a redução do erro experimental. Outra medida adotada nos ensaios 2 e 3 foi a duplicação do número de animais experimentais, utilizando-se dois galos, ISA Label (ensaio 2) ou Cobb 500 (ensaio 3), com pesos semelhantes (variação máxima de 5\%) para compor cada parcela.

No ensaio 2, a equação que descreveu o balanço de nitrogênio ( $\mathrm{mg} / \mathrm{kg}^{0,75} / \mathrm{dia}$ ) dos galos ISA Label em função do consumo de lisina digestível (mg/ $\left.\mathrm{kg}^{0,75} / \mathrm{dia}\right)$ foi: $\mathrm{BN}_{\text {ISA }}=-120,947+2,725$ CLys $\left(\mathrm{P}<0,001 ; \mathrm{r}^{2}=0,74\right)$, estimando-se as exigências de lisina para a mantença $\left(\right.$ Lys $\left._{\mathrm{mISA}}\right)$ em 44,4 mg/kg0,75/dia $(120,947 / 2,725=44,4)$. Considerando o peso médio dos galos IS A Label utilizados no ensaio 2 de 4,33 kg, as exigências corresponderam a $30,8 \mathrm{mg} / \mathrm{kg} / \mathrm{dia}\left(\left(44,4 * 4,33^{0,75}\right) / 4,33=30,8\right)$.

No ensaio 3 , a equação que descreveu o balanço de nitrogênio (mg/kg $/ \mathrm{kg}^{0,75} / \mathrm{dia}$ ) dos galos Cobb 500 em função do consumo de lisina digestível ( $\mathrm{mg} / \mathrm{kg}^{0,75} / \mathrm{dia}$ ) foi: $\mathrm{BN}_{\mathrm{C}}=-140,547+2,987$ CLys $\left(\mathrm{P}<0,001 ; \mathrm{r}^{2}=0,66\right)$, estimando-se as exigências de lisina para a mantença $\left(\mathrm{Lys}_{\mathrm{mC}}\right)$ em $47,1 \mathrm{mg} / \mathrm{kg}^{0,75} / \mathrm{dia}(140,547 / 2,987=47,1)$. Essa estimativa correspondeu a $31,3 \mathrm{mg} / \mathrm{kg} / \mathrm{dia}$, considerando o peso médio dos galos Cobb 500 de 5,11 $\mathrm{kg}\left(\left(47,1 * 5,11^{0,75}\right) / 5,11=31,3\right)$.

Os resultados comprovam que a reformulação das dietas experimentais e a duplicação do número de aves por parcela nos ensaios 2 e 3 foram medidas eficazes para reduzir o erro experimental, fato confirmado pelos menores erros-padrão (Tabela 6) e pelos maiores coeficientes de determinação $\left(\mathrm{r}^{2}\right)$ das equações obtidas nesses ensaios em relação ao ensaio com galos Leghorn.

A similaridade das respostas de balanço de nitrogênio e das estimativas das exigências, obtidas para os galos Leghorn (ensaio 1), ISA Label (ensaio 2) e Cobb 500 (ensaio 3), sugeriu a possibilidade de apenas uma equação para descrever as respostas de balanço de nitrogênio, sendo realizado um teste de paralelismo para comparar os parâmetros $\left(\beta_{0}\right.$ e $\beta_{1}$ ) das equações de cada genótipo (Kaps \& Lamberson, 2004). A ausência de diferenças $(\mathrm{P}>0,05)$ entre os parâmetros comprova que apenas uma equação foi suficiente para descrever as respostas de balanço de nitrogênio dos galos, independentemente do genótipo, descrita como: $\mathrm{BN}=-122,163+2,706 \mathrm{CLis}\left(\mathrm{P}<0,001 ; \mathrm{r}^{2}=0,67\right)$. Com base nessa equação, estimou-se a exigência de lisina para mantença de $45,1 \mathrm{mg} / \mathrm{kg}^{0,75} /$ dia (Figura 1). Considerando o peso médio geral dos galos de $3,81 \mathrm{~kg}$, as exigências corresponderam a 32,3 mg/kg/dia $\left(\left(45,1 * 3,81^{0,75}\right) / 3,81=32,3\right)$, independentemente do genótipo. 


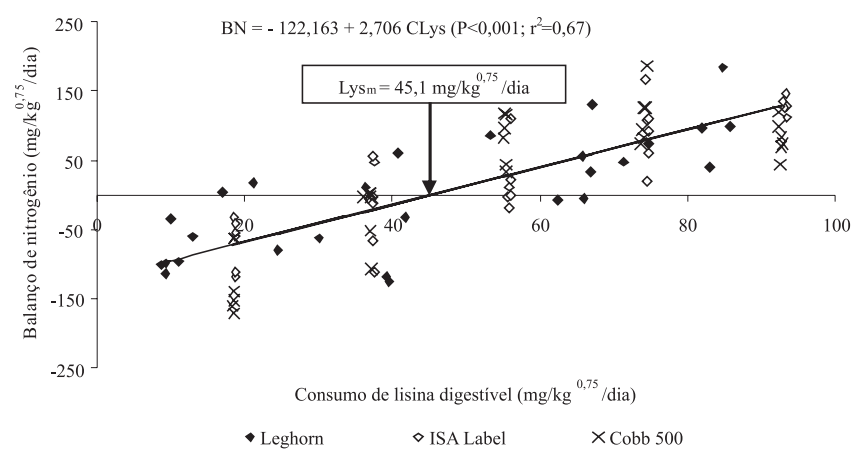

Figura 1 - Exigência de lisina digestível para mantença de galos Leghorn, Isa Label e Cobb 500 adultos.

Diferentes métodos experimentais têm sido utilizados para determinar as exigências de mantença de aves sexualmente maduras. Leveille \& Fisher (1959a) estudaram as exigências de lisina para mantença em ensaio de metabolismo com galos Leghorn utilizando dietas à base de aminoácidos cristalinos conforme método desenvolvido por Leveille \& Fisher (1958). A exigência de lisina para mantença, definida como a menor concentração de lisina nas dietas capaz de manter o balanço de nitrogênio igual a zero, foi estimada em $29 \mathrm{mg} / \mathrm{kg} / \mathrm{dia}$, equivalendo a $36 \mathrm{mg} / \mathrm{kg}^{0,75} / \mathrm{dia}$.

Sakomura \& Coon (2003) estudaram as exigências de lisina para mantença de matrizes pesadas da linhagem Cobb utilizando dietas experimentais purificadas contendo $40 \%$ das recomendações de todos os aminoácidos (NRC, 1994), com exceção da lisina, cujas concentrações variaram de $0 \mathrm{a}$ $40 \%$ das recomendações. A exigência estimada com base na relação linear entre o consumo de lisina e a deposição de lisina corporal correspondeu a 94,4 mg/kg0,75/dia.

Outra técnica foi empregada recentemente Nonis \& Gous (2008), que estudaram as exigências de lisina para a mantença de galos de linhagem de postura por meio de ensaios de metabolismo com fornecimento preciso das dietas experimentais associado à oferta ad libitum de uma dieta isenta de nitrogênio, segundo método proposto por Gous et al. (1984) e Burnhan \& Gous (1992). Foi formulada uma dieta deficiente em lisina, composta por glúten de milho e DL-triptofano, que foi intubada em diferentes quantidades de forma a proporcionar ingestões crescentes de lisina. A exigência de lisina para a mantença, estimada com base no balanço de nitrogênio, foi de $37 \mathrm{mg} / \mathrm{kg} / \mathrm{dia}$, equivalendo a $46,5 \mathrm{mg} / \mathrm{kg}^{0,75} / \mathrm{dia}$.

Neste estudo, a exigência de lisina para mantença, estimada em 45,1 mg/kg $/ \mathrm{kg}^{0,75} / \mathrm{dia}$ (32,3 mg/kg/dia), foi superior àquela obtida por Leveille \& Fisher (1959a) com galos Leghorn (36 mg/kg0,75/dia; 29 mg/kg/dia), e inferior àquela estimada por Sakomura \& Coon (2003) em experimento com matrizes pesadas $\left(94,4 \mathrm{mg} / \mathrm{kg}^{0,75} / \mathrm{dia}\right)$. Entretanto, foi muito semelhante aos resultados obtidos por Nonis \& Gous (2008) com galos adultos de linhagens de postura $\left(46,5 \mathrm{mg} / \mathrm{kg}^{0,75} / \mathrm{dia}\right.$; $37 \mathrm{mg} / \mathrm{kg} / \mathrm{dia}$ ), apesar dos diferentes métodos utilizados.

Do ponto de vista metodológico, o fornecimento de alimentação controlada pelo peso metabólico das aves possibilitou a ingestão de todo alimento ofertado, mostrando-se um método apropriado para determinar a exigência de lisina em ensaios de metabolismo com galos adultos, tendo em vista a repetibilidade dos resultados obtidos nos ensaios de metabolismo realizados neste estudo.

A exigência de lisina para mantença não variou entre os genótipos e pesos corporais quando foi estabelecida com base no peso corporal das aves. Contudo, o grau de associação entre a exigência de lisina para mantença e o peso corporal pode ser questionado, uma vez que não existe demanda de aminoácidos para manutenção das reservas lipídicas (Emmans \& Oldham, 1988; Burnham \& Gous, 1992; Gous, 2007), que podem variar consideravelmente, mesmo entre indivíduos com pesos corporais semelhantes (Emmans \& Fisher, 1986; Nonis \& Gous, 2008). Nesse sentido, para que comparações mais refinadas entre indivíduos de diferentes pesos e composições corporais seja possível, é necessário que as exigências de aminoácidos para a mantença sejam estabelecidas de modo que as diferenças no conteúdo de lipídios corporal sejam isoladas.

Com esse propósito, Emmans \& Fisher (1986), baseando-se nos estudos de Brody (1945) e Taylor \& Young (1968), relacionaram as exigências de proteína para a mantença do corpo depenado (PM; g/dia) com o peso de proteína corporal à maturidade $\left(\mathrm{PB}_{\mathrm{m}} ; \mathrm{kg}\right)$, e com o grau de maturidade da proteína corporal $\left(\mathrm{u}=\mathrm{PB}_{\mathrm{t}} / \mathrm{PB}_{\mathrm{m}}\right)$, por meio da equação: $P M=0,008 \cdot P B_{m}^{0,73} \cdot u$; em que $u=1$, por se tratarem de galos adultos $\left(\mathrm{PB}_{\mathrm{t}}=\mathrm{PB}_{\mathrm{m}}\right)$. Desse modo, as estimativas das exigências de aminoácidos para mantença foram expressas como $\mathrm{mg} / \mathrm{PB}_{\mathrm{m}}{ }^{0,73} . \mathrm{u} / \mathrm{dia}$, evitando-se os problemas inerentes às diferenças no conteúdo de lipídios corporais, conforme relatado por diversos autores (Emmans \& Fisher, 1986; Emmans \& Oldham, 1988; Burnham \& Gous, 1992; Gous, 2007; Nonis \& Gous, 2008).

Para demonstrar a aplicação da equação proposta por Emmans \& Fisher (1986), foram utilizados os dados obtidos no ensaio com galos Leghorn e os cálculos para os demais ensaios realizados da mesma forma. Considerando a estimativa de exigência de lisina para mantença de $44,9 \mathrm{mg} / \mathrm{kg}^{0,75} / \mathrm{dia}$, e o peso corporal médio de $1,99 \mathrm{~kg}$, os galos Leghorn exigiram para a mantença, em média, $75,2 \mathrm{mg}$ de lisina digestível por dia $\left(44,9.1,99^{0,75}=75,2\right)$. A concentração de proteína no corpo depenado foi 
determinada em 16\% (MN), resultando em um $\mathrm{PB}_{\mathrm{m}}$ de $0,318 \mathrm{~kg}(1,99 \cdot 16 / 100=0,318)$. Por se tratarem de aves adultas ( $\left.\mathrm{u}=\mathrm{PB}_{\mathrm{t}} / \mathrm{PB}_{\mathrm{m}}=1\right)$, a exigência de lisina digestível para mantença correspondeu à $173,6 \mathrm{mg} / \mathrm{PB}_{\mathrm{m}}{ }^{0,73} . \mathrm{u} / \mathrm{dia}$ $\left(75,2 / 0,318^{0,73} \cdot 1=173,6\right)$. Emmans \& Fisher (1986) consideraram que as aves necessitam diariamente de $8 \mathrm{~g}$ $(0,008 \mathrm{~kg})$ de proteína com composição ideal por unidade de mantença ( $\left.\mathrm{PB}_{\mathrm{m}}{ }^{0,73} \cdot \mathrm{u}\right)$. Com base nisso, a concentração de lisina na proteína ideal de mantença de galos Leghorn foi estimada em aproximadamente $22 \mathrm{~g} / \mathrm{kg}(173,6 / 8=21,6)$ (Tabela 7), confirmando a estimativa de $23 \mathrm{~g} / \mathrm{kg}$ obtida por Nonis \& Gous (2008). Esses resultados sugerem que a proporção de lisina presente na proteína para mantença difere acentuadamente daquela presente na proteína para deposição, estimada em 75 g/kg (Emmans \& Fisher, 1986; Emmans \& Oldham, 1988; Sklan \& Noy, 2004).

Apesar de não terem diferido $(\mathrm{P}>0,05)$ entre as linhagens, quando estabelecidas com base no peso metabólico ( $\mathrm{mg} / \mathrm{kg}^{0,75} / \mathrm{dia}$ ), as estimativas de exigências de lisina digestível para mantença divergiram quando expressas em relação ao conteúdo de proteína do corpo depenado, equivalendo à 173,$6 ; 164,0$ e $140,9 \mathrm{mg} / \mathrm{PB}_{\mathrm{m}}{ }^{0,73} \cdot \mathrm{u} / \mathrm{dia}$, respectivamente, para galos Leghorn, ISA Label e Cobb 500 .

Vale salientar que a exigência de lisina digestível para mantença (mg/PB ${ }_{\mathrm{m}}{ }^{0,73}$.u/dia) estimada para galos Cobb 500 foi 14\% inferior em relação aos galos ISA Label e 19\% inferior em relação aos galos Leghorn, enquanto as recomendações para galos Leghorn e ISA Label variaram apenas 5\% entre si. Partindo-se do pressuposto que não existe demanda de aminoácidos para a manutenção das reservas lipídicas (Emmans \& Fisher, 1986; Emmans \& Oldham, 1988; Burnham \& Gous, 1992; Gous, 2007; Nonis \& Gous, 2008), e considerando que, em aves de corte adultas (Cobb 500), essas reservas podem representar grandes proporções do peso corporal em relação às aves de crescimento lento (Leghorn e ISA Label), os resultados foram coerentes, evidenciando que a expressão da exigência de lisina digestível para mantença com base no conteúdo de proteína corporal pode isolar diferenças que não são detectadas quando as recomendações são estabelecidas com base no peso corporal das aves.

A expressão das exigências de lisina para mantença com base no conteúdo de proteína corporal (mg/ $\left.\mathrm{PB}_{\mathrm{m}}{ }^{0,73} \cdot \mathrm{u} / \mathrm{dia}\right)$, conforme proposto por Emmans \& Fisher (1986), possibilitou comparações mais refinadas entre aves de diferentes genótipos e pesos corporais utilizadas nos diferentes estudos. Os resultados desses estudos para a proporção de lisina presente na proteína ideal para a mantença são concordantes, e variam de 18 a $23 \mathrm{~g} / \mathrm{kg}$, confirmando que a composição da proteína ideal para mantença difere acentuadamente da composição da proteína depositada no corpo, estimada em $75 \mathrm{~g} / \mathrm{kg}$ de lisina (Emmans \& Fisher, 1986; Emmans \& Oldham, 1988; Sklan \& Noy, 2004).

O estabelecimento das exigências em unidades de mantença $\left(\mathrm{PB}_{\mathrm{m}}{ }^{0,73}\right.$.u) possibilita que as recomendações sejam extrapoladas com maior segurança para aves de diferentes genótipos e idades, por levar em consideração o conteúdo e o grau de maturidade da proteína corporal $\left(\mathrm{u}=\mathrm{PB}_{\mathrm{t}} / \mathrm{PB}_{\mathrm{m}}\right)$, que varia sistematicamente com o avançar da idade das aves.

Estimativas acuradas das exigências de aminoácidos para a mantença das aves constituem informação preponderante para a elaboração de modelos de predição das exigências com base no método fatorial. No Brasil, o uso da modelagem em nutrição de monogástricos ainda é bastante limitado e estudos que avaliaram as exigências de mantença das aves são inexistentes. Assim, surge uma diretriz para a realização de novos estudos que busquem investigar a influência do peso e da composição corporal das aves sobre os coeficientes que expressam as exigências de mantença, possibilitando a obtenção de informações que viabilizem a elaboração de modelos de predição das exigências de aminoácidos das aves.

Tabela 7 - Exigências de lisina digestível para mantença de galos adultos Leghorn, Isa Label e Cobb 500

\begin{tabular}{|c|c|c|c|c|c|c|c|}
\hline \multirow[t]{2}{*}{ Genótipo } & \multirow[t]{2}{*}{$\begin{array}{l}\text { Peso corporal à } \\
\text { maturidade }(\mathrm{kg})\end{array}$} & \multirow[t]{2}{*}{$\begin{array}{l}\text { Proteína corporal à } \\
\text { maturidade }(\mathrm{kg})\end{array}$} & \multicolumn{4}{|c|}{$\begin{array}{c}\text { Exigência de lisina } \\
\text { digestível (mg/unidade/dia) }\end{array}$} & \multirow{2}{*}{$\begin{array}{l}\text { Proteína ideal para } \\
\text { mantença }(\mathrm{g} / \mathrm{kg})\end{array}$} \\
\hline & & & Ave & $\mathrm{kg}$ & $\mathrm{kg}^{0,75}$ & $\mathrm{~PB}_{\mathrm{m}}{ }^{0,73} \cdot \mathrm{u}$ & \\
\hline 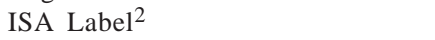 & 4,33 & 0,753 & 133,3 & 30,8 & 44,4 & 164,0 & 21 \\
\hline Cobb $500^{3}$ & 5,11 & 1,191 & 160,1 & 31,3 & 47,1 & 140,9 & 18 \\
\hline Leghorn, ISA Label e Cobb $500^{4}$ & 3,81 & 0,754 & 123,0 & 32,3 & 45,1 & 151,2 & 19 \\
\hline Leveille \& Fisher $(1959 a)^{1}$ & 2,38 & 0,381 & 69,0 & 29,0 & 36,0 & 139,6 & 18 \\
\hline
\end{tabular}

1, 2, 3, 4 Assumindo concentrações de proteína bruta (na matéria natural) de 16,0; 17,4; 23,3 e 19,8\%, respectivamente. 


\section{Conclusões}

A exigência de lisina para a mantença não varia entre aves de diferentes genótipos e composições corporais, quando é estabelecida com base no peso metabólico, sendo estimada em 45,1 mg/kg, $0075 / \mathrm{dia}$ (32,3 mg/kg/dia). Entretanto, quando são estabelecidas com base no conteúdo de proteína corporal à maturidade e no grau de maturidade da proteína corporal, as recomendações variam, especialmente entre as aves de crescimento lento e acelerado, sendo estimadas em 173,6; 164,0 e 140,9 mg/PB ${ }_{\mathrm{m}}^{0,73}$.u/dia, para galos Leghorn, ISA Label e Cobb 500, respectivamente.

\section{Agradecimentos}

Ao Conselho Nacional de Desenvolvimento Científico e Tecnológico (CNPq) pela concessão de bolsas de estudo e à Fundação de Amparo a Pesquisa do Estado de São Paulo (FAPESP) pelo financiamento da pesquisa.

\section{Referências}

ASSOCIATION OF OFFICIAL ANALYTICAL CHEMISTRY AOAC. Official methods of analysis. 16.ed. Washington, D.C.: AOAC, 1995. 2000p.

BRODY, S. Bioenergetics and growth. New York: Reinhold Publishing Animal Production, 1945. 1023p.

BURNHAM, D.; GOUS, R.M. Isoleucine requirements of the chicken: requirement for maintenance. British Poultry Science, v.33, p.59-69, 1992.

EDWARDS, H.M.; FERNANDEZ, S.R.; BAKER, D.H. Maintenance lysine requirement and efficiency of using lysine for accretion of whole-body lysine and protein in young chicks. Poultry Science, v.78, p.1412-1417, 1999.

EMMANS, G.C.; FISHER, C. Problems in nutritional theory. In: FISHER, C.; BOORMAN, K.N. (Eds.) Nutrient requirements of poultry and nutritional research. London: Butterwordths, 1986. p.9-39.

EMMANS, G.C.; OLDHAM, J.D. Modelling of growth and nutrition in different species. In: KORVER, S.; VAN ARENDONK, J.A.M. (Eds.) Modelling of livestock production systems. Amsterdam: Kluwer Academic Publishers, 1988. p.13-21.

FISHER, C. Amino acid requirements of broiler breeders. Poultry Science, v.77, p.124-133, 1998.

GONZALES, E. Ingestão de alimentos: mecanismos regulatórios In: MACARI, M.; FUNLAN, R.L.; GONZALES, E. (Eds.) Fisiologia aviária aplicada a frangos de corte. Jaboticabal: Funep, Unesp, 2002. p.187-199.

GOUS, R.M. Methodologies for modeling energy and amino acid responses in poultry. Brazilian Journal of Animal Science, v.36, p.263-274, 2007.

GOUS, R.M.; FISHER, C.; BROADBENT, L.A. Measurement of the amino acid requirement for maintenance of adult cockerels.
In: WOLRD'S POULTRY CONGRESS, 17., 1984, Helsink. Proceedings... Helsink, 1984. v.39, p.239-240.

HRUBY, M. The amino acid maintenance and growth requirements of male broilers. 1998. 144f. Thesis (Ph.D. in Animal Science) - University of Minnesota, Minnesota.

KAPS, M.; LAMBERSON, W.R. Biostatistics for animal science. Wallingford: CABI Publishing, 2004. 445p.

LEVEILLE, G.A.; SHAPIRO, R.; FISHER, H. Amino acid requirements for maintenance in the adult rooster IV. The requirements for methionine, cystine, phenylalanine, tyrosine and tryptophan; the adequacy of the determined requirements. The Journal of Nutrition, v.72, p.8-15, 1960.

LEVEILle, G.A.; FISHER, H. Amino acid requirements for maintenance in the adult rooster II. The requirements for glutamic acid, histidine, lysine and arginine. The Journal of Nutrition, v.69, p.289-294, 1959a.

LEVEILLE, G.A.; FISHER, H. Amino acid requirements for maintenance in the adult rooster III. The requirements for leucine, isoleucine, valine and threonine, with reference also to the utilization of the d-isomers of valine, threonine and isoleucine. The Journal of Nutrition, v.70, p.135-140, 1960.

LEVEILLE, G.A.; FISHER, H. The amino acid requirements for maintenance in the adult rooster. The Journal of Nutrition, v.66, p.441-452, 1958.

LEVEILLE, G.A.; FISHER, H. The amino acid requirements for maintenance in the adult rooster: Nitrogen and energy requirements in normal and protein-depleted animals receiving whole egg protein and amino acid diets. Journal of Nutrition, v.66, p.442-453, 1959b.

NATIONAL RESEARCH COUNCIL - NRC. Nutrient requirements of poultry. 9.ed. Washington: National Academy of Science, 1994. 176p.

NONIS, M.K.; GOUS, R.M. Threonine and lysine requirements for maintenance in chickens. South African Journal of Animal Science, v.38, p.75-82, 2008.

OVIEDO-RONDÓN, E.O.; WALDROUP, P.W. Models to estimate amino acid requirements for broiler chickens: a review. International Journal of Poultry Science, v.5, p.106-113, 2002 .

OWENS, F.N.; PETTIGREW, J.E. Subdividing amino acid requirements into portions for maintenance and growth. In: FRIEDMAN, M. Absorption and utilization of amino acids. Boca Raton: CRC Press, 1989. v.1, p.15-30.

ROSTAGNO, H.S.; ALBINO, L.F.T.; DONZELE, J.L. et al. Tabelas brasileiras para aves e suínos: composição de alimentos e exigências nutricionais. 2.ed. Viçosa, MG: Departamento de Zootecnia, Universidade Federal de Viçosa, 2005. 186p.

SAKOMURA, N.K.; COON, C. Amino acid requirements for maintenance of broiler breeder pullets. In: EUROPEAN SYMPOSIUM ON POULTRY NUTRITION, 14., 2003, Lillehammer. Proceedings... Lillehammer, 2003. p.280-281.

SAKOMURA, N.K.; ROSTAGNO, H.S. Métodos de pesquisa em nutrição de monogástricos. Jaboticabal: Funep, 2007. 283p.

SKLAN, D.; NOY, Y. Catabolism and deposition of amino acids in growing chicks: effect of dietary supply. Poultry Science, v.83, p.952-961, 2004.

TAYLOR, C.S.; YOUNG, G.B. Equilibrium weight in relation to food intake and genotype in twin cattle. Animal Production, v.10, p.393-412, 1968. 